\title{
A bipolar LED drive technique for high performance, stability and power in the nanosecond time scale
}

\author{
E. Ronchi ${ }^{\mathrm{a}, *}$, E. Andersson Sundén ${ }^{\mathrm{a}}$, S. Conroy ${ }^{\mathrm{a}}$, G. Ericsson ${ }^{\mathrm{a}}$, M. Gatu Johnson ${ }^{\mathrm{a}}$, J. Kallne ${ }^{\mathrm{a}}$, C. Hellesen $^{\mathrm{a}}$, \\ A. Hjalmarsson ${ }^{a}$, H. Sjöstrand ${ }^{a}$, M. Weiszflog ${ }^{a}$, W. Puccio ${ }^{b}$, L. Åhlén ${ }^{b}$, JET-EFDA Contributors ${ }^{\mathrm{c}, 1}$ \\ a Department of Physics and Astronomy, Uppsala University, 75120 Uppsala, Sweden ${ }^{2}$ \\ ${ }^{\mathrm{b}}$ Department of Astronomy and Space Physics, Uppsala University, 75120 Uppsala, Sweden \\ c JET-EFDA, Culham Science Center, OX14 3DB, Abingdon, UK
}

\section{A R T I C L E I N F O}

\section{Article history:}

Received 17 July 2008

Received in revised form 23 October 2008

Accepted 3 November 2008

Available online 19 November 2008

\section{Keywords:}

LED

Driver

Nanosecond

Fast

Bipolar

High stability

High power

JET

MPRu

TOFOR

\begin{abstract}
A B S T R A C T
Pulsed light sources are often used to monitor the stability of light detectors such as photomultiplier tubes. Light emitting diodes (LEDs) are suitable for this due to their high specific light yield. While pulsed operation in the region of $\mu$ s is generally accessible with most LEDs and drivers, the ns time scale often represents a technical challenge. This paper describes a technique of bipolar LED drive that can produce light pulses of a few ns at high stability, reliability and power. The driver also offers control over the properties of the light pulse produced such as shape, intensity and repetition rate.

This approach has been studied in 2003 and implemented in 2004 for two fusion neutron spectrometers at the Joint European Torus (JET) namely the Magnetic Proton Recoil upgrade (MPRu) and the Time Of Flight Optimized for Rate (TOFOR). A driver has been manufactured and connected to the scintillation detectors of each spectrometer through an optical fiber distribution network. Both MPRu and TOFOR have been successfully relying on this system for calibration and performance monitoring for several years, confirming the long-term stability and reliability of this technique.
\end{abstract}

(c) 2008 Elsevier B.V. All rights reserved.

\section{Introduction}

Light sources are commonly used for calibrations of detectors such as photomultiplier tubes (PMT) and for monitoring their stability over time. An absolute reference (primary standard) can be provided by weak long-lived radioactive sources coupled to scintillation detectors. However, in cases when one wishes to distribute the same signal over an array of detectors, a more powerful secondary standard is required. Light emitting diodes (LEDs) [1] represent a valid choice for this purpose due to their high light yield per unit surface and the relatively simple electronic drivers they require. The main limitation in using LEDs comes from the minimum pulse duration attainable which is due to the inherent properties of the junction of the LED. Obtaining pulses as short as a few ns represents a technical challenge

\footnotetext{
*Corresponding author at: Angstromlaboratoriet, 75120 Uppsala, Sweden. Tel: +46184712797.

E-mail address: emanuele.ronchi@tsl.uu.se (E. Ronchi).

${ }^{1}$ See the Appendix of M.L. Watkins et al., Fusion Energy 2006 (Proc. 21st Int. Conf. Chengdu, 2006) IAEA, (2006).

${ }^{2}$ EURATOM-VR Association.
}

especially if high output power is required. Simple drivers often make use of a single transistor stage to inject current directly into the LED anode [2]. This approach yields good results but is not generally suitable for the nanosecond time scale due to the parasitic capacitance of the diode. In these conditions the light signal was found to extend significantly beyond the current pulse; this is here referred to as afterglow. Fig. 1 shows an example of afterglow of a high-power LED of $7 \mathrm{~mm}$ diameter [3] in response to a $10 \mathrm{~ns}$ current pulse produced through a simple transistor stage. A fast PMT suitable for light pulses as short as few ns was used for detection. It can be seen that the tail extends several $\mu$ s after the peak.

\section{Design of a bipolar LED driver}

A bipolar technique has been developed to limit the afterglow and achieve nanosecond performance as shown in Fig. 2. This is composed of two distinct traces. The upper one (black) is applied to the LED anode, and the other (gray) to the cathode. It can be seen that the LED is subjected to a weak forward bias (FB) prior to the pulse, intended to optimize the speed of the off-to-on 


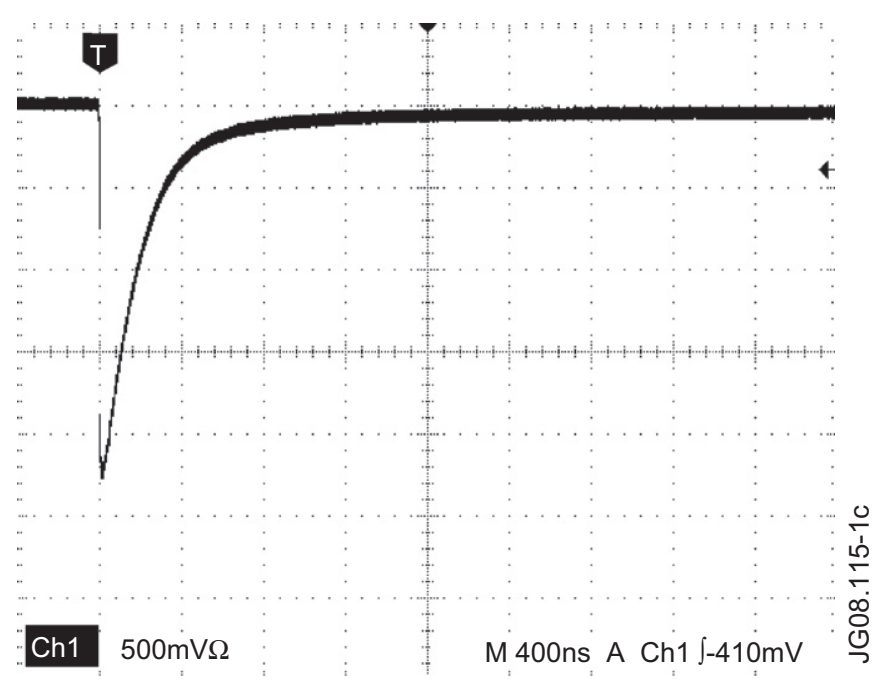

Fig. 1. Example of pulse and afterglow with a simple transistor LED drive (horizontal scale: $400 \mathrm{~ns} /$ div, vertical scale: $500 \mathrm{mV} /$ div).

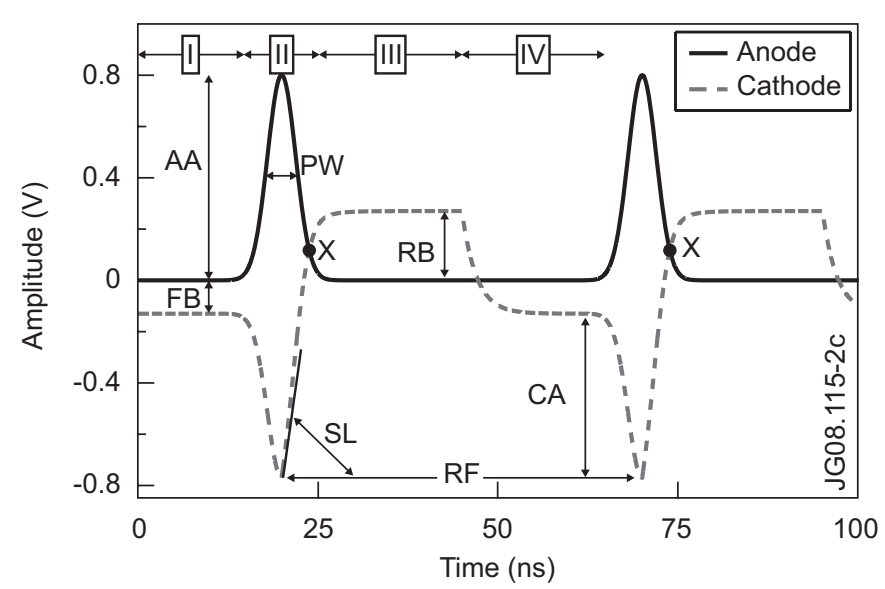

Fig. 2. The principle of the bipolar LED drive technique. Anode (black) and cathode (gray) traces shown.

transition (phase I). The current pulse is generated in a differential fashion. This enhances the light yield from the diode through an electrical push-pull effect from both electrodes (phase II). The bias is then continuously reversed in the following stage (phase III) causing a controlled depletion of the electrical charge in the junction. The forward polarization is then restored in the last phase (phase IV) which leads again to I and restarts the cycle. The bipolar approach offers control over a number of parameters regulating the LED transition times, the shape of the emitted light pulse and its intensity. The electronic implementation of these is illustrated in Fig. 3.

The FB in phase I is marked as FB and is regulated through the resistor R23 in Fig. 3. FB should always lie below the LED threshold if continuous light from the device is to be avoided. Phase II includes two controls to independently regulate the anode and cathode pulse amplitudes, AA and CA, through resistors R4 and R5, respectively. Higher AA and CA settings produce a larger current in the diode, yielding greater light intensity. Phase III includes two parameters as well, namely SL (resistor R10) and reverse bias (RB) (resistor R8) in the figure. By varying the slope (SL) of the cathode trace it is possible to move the intersection point (X-point) with respect to the peak. Higher SLs cause the $\mathrm{X}$-point to approach the peak and to reverse the LED polarity sooner after the pulse. The amplitude of the RB has an additional effect on the rate of the charge depletion at the junction, actively shaping the light pulse trailing edge. Proper settings of SL and RB are essential to shape the pulse or to achieve the fastest possible response from each diode. This is discussed in further detail in Section 3. Finally, pulse width (PW, resistor R1) and repetition frequency $(\mathrm{RF})$ can be set to suit the application. The latter is regulated from a separate section not shown in Fig. 3 connected to the onboard voltage-controlled oscillator [4]. The entire cathode trace can be time-shifted with respect to the anode peak, providing an additional control for fine-tuning the output (resistor R26).

The current implementation of the driver can support up to three LEDs and a fast NIM-compatible output for timing measurements. The latter is marked as "SYNC OUT" in Fig. 3 and is intended to provide a reference signal each time a LED pulse is generated. The LED can either be connected directly at the output stage of Fig. 3 with an appropriate resistor as current limiter or can be installed on a separate circuit board (LED board). The latter is the configuration of choice for optimal performance and power and uses $50 \Omega$ coaxial cables and terminators on both sides. In the present configuration two operational amplifiers have been installed on the LED board to electrically decouple the diode from the driver avoiding direct load on the unit. The LED current is controlled by a single high-frequency potentiometer, as shown in Fig. 4.

The components and the circuit board layout used for the driver are of high-frequency type in order to minimize the total parasitic capacitance and enhance the bandwidth of the signal [5]. The amplifiers OPA3692 by Texas Instruments [6] have been chosen for the driver. These have proven to be a good compromise between speed, stability and pulse response characteristics. The amplifiers OPA695 [7] were chosen for the LED board and for the summing node of the driver (IC5A in Fig. 3). Despite not featuring the widest bandwidth available, the OPA695 was found to be very reliable and stable for pulse amplification and capacitive load drive. Current sourcing and sinking capabilities are high for DC $(120 \mathrm{~mA})$ but laboratory tests have demonstrated that these amplifiers can reliably source or sink up to $1 \mathrm{~A}$ at low duty cycles (e.g. a few ns pulses at $1-1000 \mathrm{kHz}$ repetition rate). All resistors are surface-mounted and the potentiometers are of the lowinductance cermet type. The generation of electrical pulses of a few ns is achieved using Advanced Ultra-low-voltage CMOS discrete logic gates [8] and a high-frequency printed circuit board layout. In particular, a two-layer design was sufficient for the task.

\section{Characterization of performance and results}

The bipolar driver has been tested with several LEDs on detectors of relevance for the MPRu and TOFOR fusion neutron spectrometers [9-11]. The detectors used are PMT of type MPRu, TOFOR/S1 and TOFOR/S2 [12]. The determination of the shortest attainable light pulse from a given diode is of interest to assess the performance of the bipolar approach in the nanosecond time scale. A MPRu PMT was used here due to its fast response and a small $1.8 \mathrm{~mm}$ surface-mounted LED [13] was chosen for these tests. The LED is of the general-purpose type and it is not specifically designed for nuclear applications. Nevertheless, it features low junction capacitance and high-power density. Furthermore, its dominant wavelength $(466 \mathrm{~nm})$ is similar to that of the light produced by the scintillation detectors currently installed in MPRu and TOFOR (i.e. 405 and $395 \mathrm{~nm}$ respectively) and lies near the quantum efficiency peak of the related PMTs.

In this test, the diode is interfaced to the PMT through a thin $100 \mu \mathrm{m}$ optical fiber. A $2.5 \mathrm{G}$ sample/s ADC is used to digitize the waveforms and to store them for data analysis. The pulse duration 


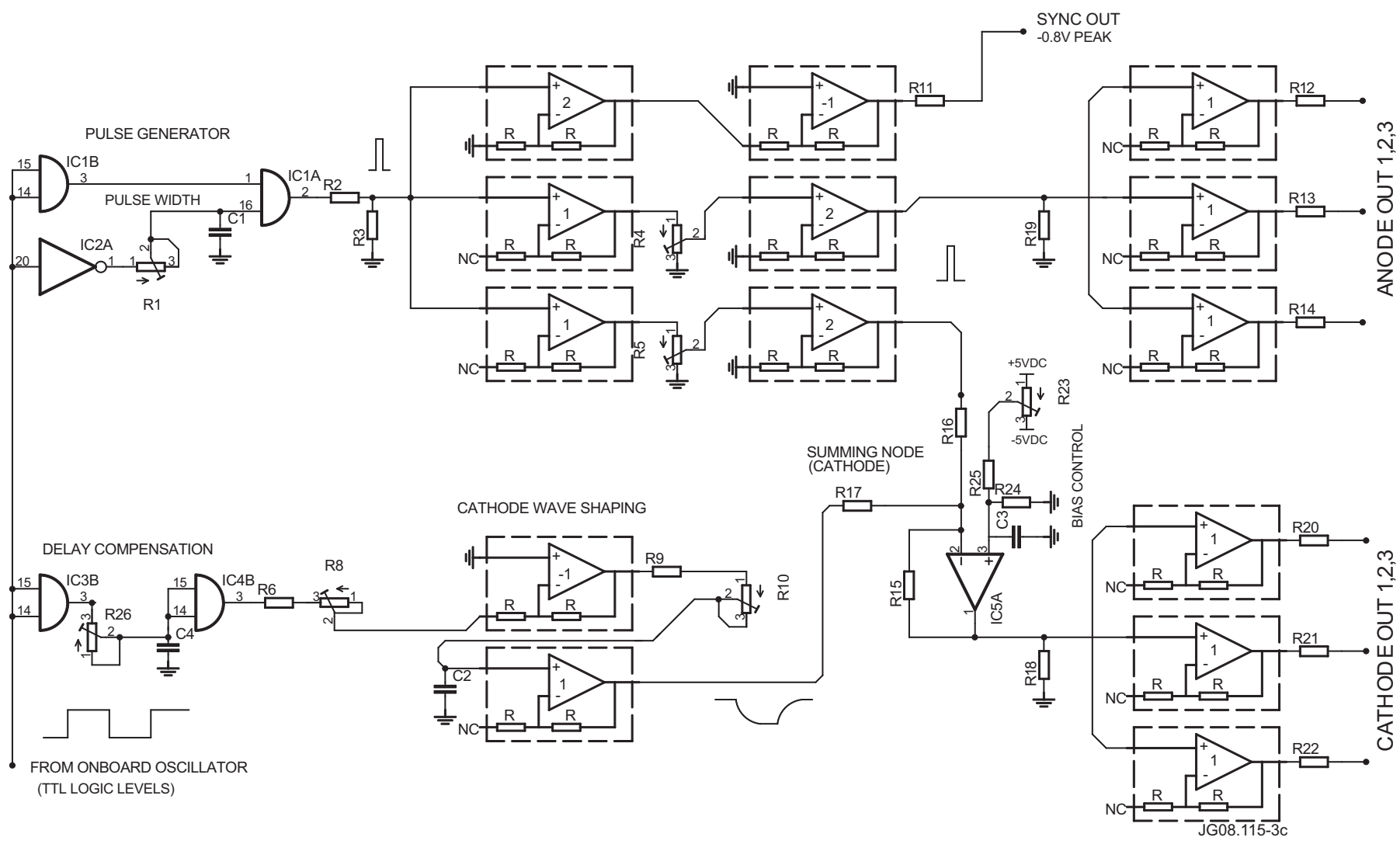

Fig. 3. Electronic implementation of the bipolar LED driver.

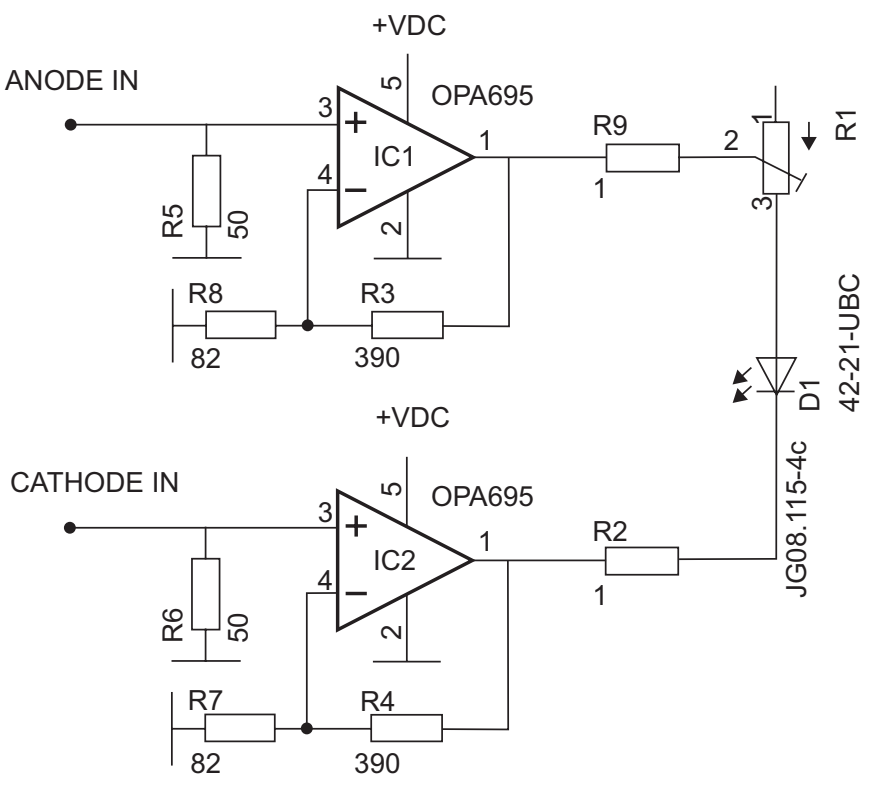

Fig. 4. The LED board electronic layout.

at half height $(\Delta t)$ has been studied at various parameter settings. It has been found that $\Delta t$ responds to the PW control almost linearly for pulse duration above $10 \mathrm{~ns}$ but shows a saturation effect when $\Delta t$ is reduced below this limit. From 10 to $6 \mathrm{~ns}$ it is no longer possible to act only on PW. It becomes necessary to modify the FB and increase the amplitude of the anode pulse. Also the cathode wave needs to be modified by increasing SL and RB. Finally, the current limiter in the LED board must be reduced as well to match the requirements. The width reduction becomes

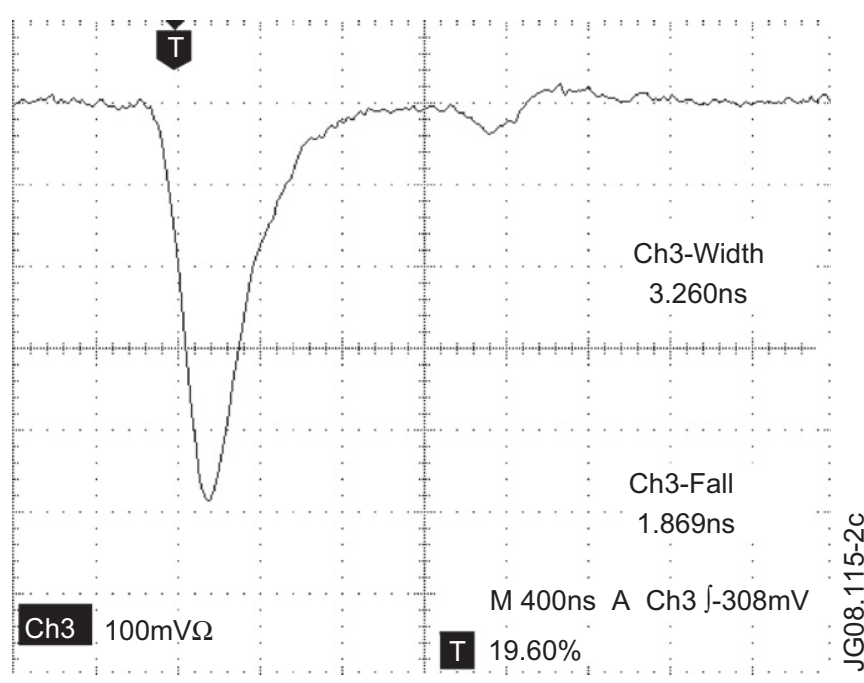

Fig. 5. The shortest light pulse obtained with bipolar driver using a $1.8 \mathrm{~mm}$ LED (Everlight 42-21UBC/C430/TR8) and an MPRu-type PMT (horizontal scale: 4 ns/div, vertical scale: $100 \mathrm{mV} /$ div).

more difficult below 6 ns until the diode stops responding to the controls when the duration reaches approximately 3 ns. Fig. 5 shows the minimum PW attainable from the $1.8 \mathrm{~mm}$ LED under these experimental conditions. The onset of a barrier towards progressively smaller pulse durations is expected and depends on several factors such as the junction capacitance of the LED, the board components and the printed circuit board design. Furthermore, the PMT itself constitutes another limiting factor since the nominal minimum $\Delta t$ from the manufacturer 
$(\Delta t \sim 2.7-3 n s)$ is very close to the pulse duration shown in Fig. 5. More accurate assessment of the minimum light pulse duration would require a faster detector capable of sub-ns performance.

Stability tests of the device have been performed by monitoring the LED light amplitude versus time (Fig. 6a). Here the output is normalized to the mean value and plotted versus time during a test period of $15 \mathrm{~h}$. The light signal duration has been set to $3.5 \mathrm{~ns}$ on the $1.8 \mathrm{~mm}$ diode and the intensity at roughly $90 \%$ of the maximum ratings of the LED and driver. A fiber optic attenuator has been included to prevent PMT damage. This test is meant to record the stability of the system under challenging conditions of pulse duration and power output. Fig. $6 \mathrm{~b}$ provides a statistical interpretation of the data and the results are summarized in Table 1 . The variations in the output over time result in the broadening of the distribution of less than $0.2 \%$. The small secondary peak found at about $99.65 \%$ is likely due to small temperature variations during the test. Other tests did not show peaks at this particular position. Further tests could be performed at a rigorously constant room temperature to filter out any temperature dependence.

\section{Discussion}

The bipolar approach does not have a theoretical limit in the width of the current pulse produced. The current is drawn into the diode when the difference of the two traces exceeds a threshold and this region can be made arbitrarily small by controlling the time offset between the two signals, increasing SL and reducing PW or changing FB. This means that the main limiting factor lies in the physical characteristics of the LED substrate. This statement was confirmed when the driver was tested on other diodes. In particular, the $7 \mathrm{~mm}$ high-power device [3] featured a minimum PW of 18-20 ns. This is about six times longer than the one of the $1.8 \mathrm{~mm}$ diode but still considerably shorter than with the standard transistor drive, as shown in Fig. 1. When approaching the limit conditions of duration and power, a small damped oscillation has been observed after the main peak, as illustrated in Fig. 5. This is likely an effect of the limiter resistor (R1 in Fig. 4) which has been reduced to very low values for maximizing the total current in the diode. The onset of oscillations when driving capacitive loads with

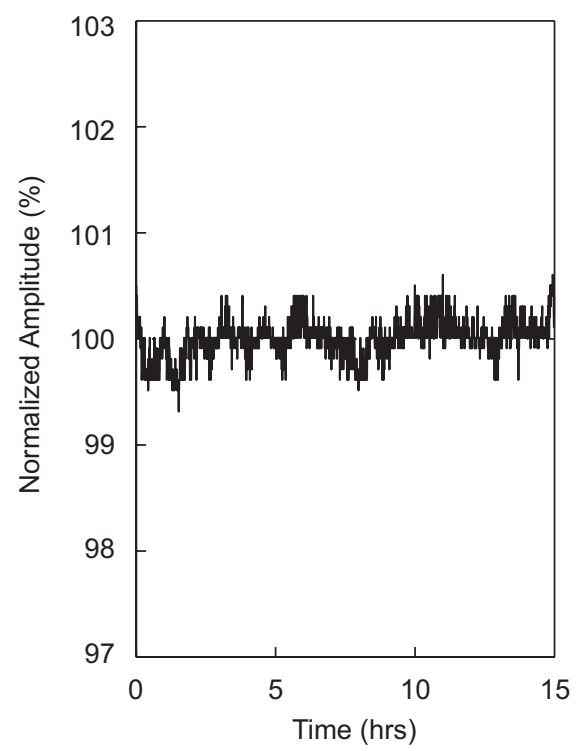

operational amplifiers was foreseen at high frequency and at low values of R1. Several configurations have been tested for the LED board. However, the one presented here yielded the best performance despite this effect. There have been no indications that this is a source of short or long-term instability for the driver.

Logic components have been used instead of analog devices for the generation of the pulse. This was primarily due to their fast transition speed. In particular, the AND gate (IC1A in Fig. 3) allows for logic switching (e.g. 10-90\%) on the order of 1-2 ns. However, the full $0-100 \%$ transition takes $1-2$ ns longer to complete and the finite slew rate of the amplifiers adds further delay. This means that if PW is set below 5-6 ns, the AND gate switches back during the pulse rise time. This has a direct impact on the amplitude, which decreases. Although this can be compensated through the voltage divider R2-R3, the coupling between PW and amplitude is generally not desirable. This could be improved through the use of faster logic gates and amplifiers not available at the time of the development described here.

Temperature dependence has not been studied in detail during the development of the driver since the device was meant to operate in a temperature-controlled environment. However, laboratory tests have highlighted a small positive temperature correlation of the integrated charge of the anode and cathode traces. This is approximately $+0.1-0.2 \% /{ }^{\circ} \mathrm{C}$ for pulses above $5-6 \mathrm{~ns}$. This effect increases for pulses shorter than $5 \mathrm{~ns}$ due to the width-amplitude coupling discussed above. Temperature drifts can reach up to $+2-3 \% /{ }^{\circ} \mathrm{C}$ in extreme cases, especially if $\mathrm{PW}$ is at minimum ( $\mathrm{R} 1=0$ in Fig. 3 ) and the bias always reversed $(\mathrm{FB}<0$ through R23). Constant-temperature stability and reliability of the device, however, remained in line with Fig. $6(\sim 0.1-0.2 \%)$ under all operating modes.

Table 1

Main parameters of the statistical distribution of the LED normalized amplitude.

\begin{tabular}{lc}
\hline & Value (\%) \\
\hline Minimum & 99.299 \\
Maximum & 100.559 \\
Mean value & 100.000 (by default) \\
Most probable value & 99.992 \\
Standard deviation & 0.176 \\
\hline
\end{tabular}

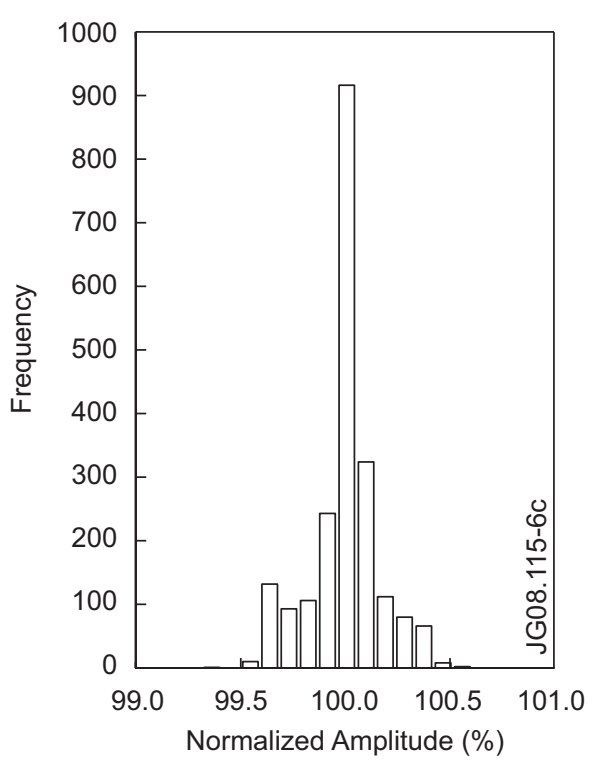

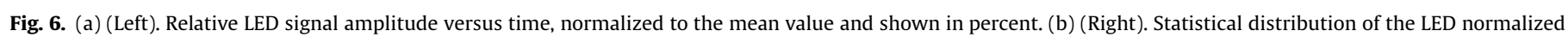
amplitude, in percent. 
In general, a positive temperature correlation is useful in a LED driver since it can partly compensate the known negative thermal coefficient of the diode [14]. However, the large number of controls in the driver makes it difficult to match the two contributions for all cases. Feedback control including optical and temperature sensors is advisable for operation in environments with large temperature variations. Alternatively a yieldtemperature curve can be measured to compensate the thermal drifts.

\section{Conclusion}

The bipolar approach has proven to be effective for driving LEDs of a variety of sizes and power. It exploits the LED characteristics to their full extent and allows operations on short time scales not generally accessible with simpler implementations. This technique also provides the possibility to shape the trailing edge of the light pulse, although with some limitations. This is of interest in applications of pulse shape analysis and scintillation detector tests, where the light pulse can be set to mimic the signal produced by a particular combination of scintillator and radioactive source. Experimental results obtained during the development phase showed that the bipolar technique guarantees a high level of stability, here found to be $0.1-0.2 \%$ in a temperature-controlled environment. The found stability and the possibility of varying the pulse repetition frequency and its shape make the device suitable for gainrate studies of radiation detectors as well as for detector monitoring.

The bipolar concept has been developed and implemented for the MPRu and TOFOR fusion neutron spectrometers in 2003 and deployed at JET during fall 2004. Up to the present, data collected on-site validates the results presented in this paper [15]. Additionally, it also proves long-term stability and reliability of such approach over the first few years of operation.

\section{Acknowledgments}

This work has been performed under the European Fusion Development Agreement (EFDA) and the Association EURATOMVR with support from Swedish Research Council (VR), Uppsala University and JET-EFDA. The views and opinions expressed herein do not necessarily reflect those of the European Commission.

\section{References}

[1] Light Emitting Diode. Available online at $\langle$ http://en.wikipedia.org/wiki/LED $\rangle$.

[2] A.S. Sedra, K.C. Smith, Microelectronic Circuits, fourth ed., Oxford University Press, USA, 1997, 1360pp (ISBN-10: 0195116631, ISBN-13: 978-0195116632).

[3] LXHL-NRR8 Luxeon Star/O-Royal Blue Batwing LED. Datasheet available at 〈http://www.luxeonstar.com/item.php?id=368\&link_str=\&partno=LXHL-NRR8 $\rangle$.

[4] Texas Instruments SN74LS628N voltage-controlled oscillator. Datasheet available at $\langle$ http://focus.ti.com/lit/ds/symlink/sn74ls628.pdf $\rangle$.

[5] J.C. Blankenhorn, PCB Design of High Speed Circuits, 2002 (ISBN: 1-882812-04-2).

[6] Texas Instruments OPA3693 triple, ultra-wideband, fixed-gain, video buffer with disable. Datasheet available at <http://focus.ti.com/lit/ds/symlink/ opa3693.pdf $>$.

[7] Texas Instruments OPA695 ultra-wideband, current-feedback operational amplifier with disable. Datasheet available at <http://focus.ti.com/lit/ds/ symlink/opa695.pdf $>$.

[8] AUC Advanced Ultra-low-voltage CMOS Data Book, Texas Instruments, 2003. Available online at 〈http://focus.ti.com/lit/ug/sced011a/sced011a.pdf $\rangle$.

[9] G. Ericsson, et al., Rev. Sci. Instrum. 72 (2001) 759.

[10] M. Gatu Johnson, et al., Nucl. Instr. and Meth. Phys. Res. A 591 (2008) 417.

[11] A. Hjalmarsson, et al., Characterization of a scintillator detector with charged particles and pulse light emission, UU-NF 05\#13 Uppsala University, Neutron Physics Report, 2005, (ISSN 1401-6269).

[12] ElectronTubes JET_A_PV19VN-06, JET_B_P30VN-06, JET_C_P61VN-01 custom photomultiplier tubes. Details available upon request online at $\langle$ http://www. et-enterprises.com/ $>$.

[13] Everlight 42-21UBC/C430/TR8 Chip LED with $1.8 \mathrm{~mm}$ round subminiature. Datasheet available at <http://www.everlight.com/upload/product_pdf/ 42-21UBC-C430-TR8_858.pdf $\rangle$.

[14] C. Huh, W.J. Schaff, L.F. Eastman, S.-J. Park, Temperature dependence of performance of InGaN/GaN MQW LEDs with different indium compositions, Electron Device Letters, IEEE, 25(2) (2004) 61-63, Digital Object Identifier 10.1109/LED.2003.822659.

[15] H. Sjöstrand, et al., Control and monitoring system of the upgraded magnetic proton recoil neutron spectrometer at JET, 2008, EFDA-JET preprint EFDA-JETPR(08)07. 